\title{
Three-Fermion Bound States on the Light Front
}

\author{
Stefano Mattiello, Stefan Strauss \\ Institut für Theoretische Physik, Universität Gießen, D-35390 Gießen, Germany
}

\begin{abstract}
We investigate the stability of the relativistic three-fermion system with a zero-range force in the light front form. In particular, introducing an invariant cut-off, we study the dependence of the bound state on the coupling strength also for cases where the two-fermion system is unbound. The relativistic Thomas collapse is discussed by solving the fully coupled integral equation system. Furthermore, we explicitly investigate the ground state mass of the three-fermion system and compare to previous simplified calculations.
\end{abstract}

Key words: three-body equation, Light front, stability

PACS: 12.39.Ki 21.45.-v 11.10.St

\section{Introduction}

Relativistic constituent quark models in light front dynamics have received much attention lately [1]. A major advantage of this framework is the covariant description of few-body bound states under kinematical front form boosts [2]. This is a consequence of the stability of the light front Fock state decomposition under boost transformations [3]. Several studies have addressed nucleon properties, such as electromagnetic form factors, using quarks as the relevant degrees of freedom and Gaussian wave functions on the light front $[4,5,6]$. Other calculations of the proton electric form factors [1] use nucleon null-plane wave functions obtained from the solution of the Faddeev equation [7] with a zero-range force [8] acting between the constituent quarks. In this effective model the spin degrees of freedom and confinement are missing. Naturally, zero-range interactions provide a simple, but important limiting case for short range forces and there has been renewed interest in zero-range models with applications in nuclear and atomic physics 
in the past years $[9,10,11,12]$. The present work is an extension of the developments given in Ref. [8], where the three-boson bound state problem on the light front was considered. Subsequently, the relativistic three-boson problem with zero-range interactions using light front dynamics has been reformulated by Carbonell and Karmanov in a covariant way [11]. While in the former publication the zero-range interaction was smeared, the authors of [11] only assumed the existence of a two-body bound state for the UV regularization without introducing further restrictions in the Faddeev equation itself. With the latter regularization scheme, which is called $M_{2 B}$-regularization in the following, the mass of the three-body bound state may vanish despite the finite value of the mass of the bounded two-body system. This is the relativistic analog of the so-called Thomas collapse. Originally, it was pointed out that non-relativistic three-body systems, based on zero-range forces, experience a Thomas collapse [13]. Since the inverse of the binding energy $\left|B_{3}\right|^{-1}$ is a measure of the size of the system the three-body state collapses when the binding energy is unbounded from below, i.e. $B_{3} \rightarrow-\infty$.

A flaw of the $M_{2 B}$-regularization scheme, as used in [11], is that the explicit dependence of the three-body mass on the coupling constant is not recovered, since the three-body bound state mass is parameterized by the two-body mass. In order to investigate the three-body bound state equation even if no two-body bound state exists, the introduction of a different regularization scheme is necessary. To this end an invariant cut-off $\Lambda$ as proposed by Lepage and Brodsky in [14] was introduced into the three-boson equation in Ref. [15]. More specifically, the requirement is that the masses of the twoand three-body systems are smaller than the cut-off, i.e. $M_{20}^{2}, M_{30}^{2}<\Lambda^{2}$. It has been shown in Ref. [15] that in the Lepage-Brodsky (LB) regularization scheme the limit of very large $\Lambda$ coincides with the results of Carbonell and Karmanov where a comparison can be made. In particular, the relativistic Thomas collapse appears for any value of $\Lambda$.

The validity of the bosonic model is evident by the calculation of the proton electric form factor obtained from the Faddeev wave functions, that reproduces well the experimental data for low momentum [1]. The dominance of this scalar channel for the description of the nucleon static properties has been confirmed by systematic investigations of the relativistic quark coupling effects in the nucleon electromagnetic form factors [16]. The scalar coupling between the quark-pair is preferred by neutron data, independently on the choice of the momentum wave functions [17, 18, 19].

However, to achieve a more realistic description of the nucleon the spin 
degrees of freedom have to be accounted for. This leads to a coupled system of integral equations instead of one Faddeev equation. The first step in this direction has been done again by Karmanov and Carbonell [20], where the first results using the $M_{2 B}$ - regularization for a two-fermion kernel have been reported. The well-known difficulties to treat the spin on the light front can be reduced by using kernels factorized relative to initial and final states. In particular, Karmanov and Carbonell have chosen a simplified kernel with properties similar to the bosonic interaction. Indeed, the successes of the bosonic model describing the proton properties indicate that this limitation is reasonable.

In this paper we revisit the interaction given in [20] using the LB regularization scheme. In doing so we relax their limitations and investigate

(i) the three-fermion equation even if no two-fermion subsystem exist,

(ii) the direct dependence of the two- and three-body bound states on the coupling strength,

(iii) in contrast to [20], the full set of coupled integral equations and

(iv) the critical coupling for the Thomas collapse.

This paper is organized as follows. In the Section 2 we present the derivation for the two- and three-fermion bound state equation on the light front using a general zero-range interaction where the kernel is factorized relative to the initial and final states. We consider a specific interaction kernel corresponding to a ${ }^{1} S_{0}$ nucleon in the Section 3 and derive the coupled integral Faddeev equations. Our results are compared to the previous calculations of Carbonell and Karmanov and to the three-boson case in the Section 4. A discussion of the main findings and perspectives is given in the conclusions.

\section{2-Fermion and 3-Fermion bound states for separable kernels}

The zero-range two-fermion kernel can be constructed utilizing different spin couplings. We use kernels being factorized relative to initial and final states, thus having the form

$$
\mathcal{K}_{\sigma_{1} \sigma_{2}}^{\sigma_{1}^{\prime} \sigma_{2}^{\prime}}\left(1,2 ; 1^{\prime}, 2^{\prime}\right)=\lambda \bar{K}_{\sigma_{1} \sigma_{2}}(1,2) K^{\sigma_{1}^{\prime} \sigma_{2}^{\prime}}\left(1^{\prime}, 2^{\prime}\right)
$$

with

$$
\bar{K}_{\sigma_{1} \sigma_{2}}(1,2) \propto\left[\bar{u}_{\sigma_{1}}\left(p_{1}\right) i \psi \bar{V} \bar{u}_{\sigma_{2}}\left(p_{2}\right)\right]
$$




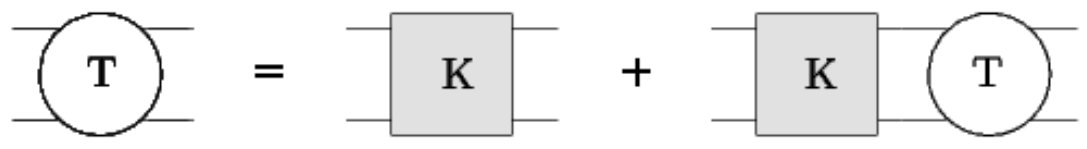

Figure 1: Equation for the two-body $t$-matrix with zero-range interaction.

where $\bar{V}$ indicates the interaction vertex, $\sigma_{i}$ denote the spin indices of the particles and $u_{\sigma_{i}}$ the corresponding spinors. The factorization (of the kernel) leads to simplifications that are independent from the specific form of the spin coupling.

\subsection{The two-body problem}

We briefly review the basic ingredients of the two-body equations on the light front given previously in $[1,8,15]$. For a zero-range interaction the equation represented by Fig. 1 can be summed leading to a solution for the two-particle propagator $t\left(P_{2}\right)$, i.e.

$$
t\left(P_{2}\right)=\left(i \lambda^{-1}-B\left(P_{2}\right)\right)^{-1},
$$

where $P_{2}^{\mu}$ is energy-momentum vector of the two-body system. The expression for $B\left(P_{2}\right)$ corresponds to a loop diagram and is given by

$$
B\left(P_{2}\right)=-\frac{i}{2(2 \pi)^{3}} \int \frac{d x^{\prime} d^{2} k_{\perp}^{\prime}}{x^{\prime}\left(1-x^{\prime}\right)} \frac{K}{P_{2}^{2}-M_{20}^{2}},
$$

where

$$
\begin{aligned}
M_{20}^{2} & =\left(\vec{k}_{\perp}^{2}+m^{2}\right) / x^{\prime}\left(1-x^{\prime}\right) \\
K & =\lambda^{-1} \operatorname{Tr}\{\mathcal{K}\} .
\end{aligned}
$$

$M_{20}$ is the mass of the virtual two-particle state and $m$ the mass of the constituent particles. The integration variables in the loop are $x^{\prime}, \vec{k}_{\perp}^{\prime}$ are the relative coordinate of the two-body system as defined in Appendix A. Equation (4) is a generalization of the corresponding equation in the bosonic case $[1,8,15]$, where the spin structure has been missing. This which is equivalent to $K=1$ in eq. (4). The integral (4) has for a contact interaction an UV divergence that has to be regularized. If the kernel leads to a logarithmic divergence, like for the scalar bosons, it can be absorbed in a 
redefinition of $\lambda$ by assuming that the two-particle propagator $t\left(P_{2}\right)$ has a pole at $P_{2}^{2}=M_{2 B}^{2}$. Then holds the relation

$$
i \lambda^{-1}=B\left(M_{2 B}\right) \text {. }
$$

Hence, the renormalization scale of the propagator is fixed by the mass of the two-particle $M_{2 B}$. With the $M_{2 B}$-regularization the condition (7) imposed in the denominator of eq. (3), i.e. $B\left(M_{2 B}\right)-B\left(P_{2}\right)$, cancels the UV divergency of $t\left(P_{2}\right)$. The assumption of the existence of a two-body bound state regularizes the loop integral completely only for a logarithmic divergence. For completeness, we remark that, in general, additional conditions have to be introduced in order to fully regularize the self-energy $B\left(P_{2}\right)$. Note, that using the $M_{2 B}$-regularization scheme the relation between the two-body mass and the coupling constant is not resolved. The two-particle propagator is the input for the relativistic three-body equations. In order to investigate the three-body bound state equation also for cases where no two-body bound state exists another regularization scheme has to be used. This allows us to find the relation between the coupling constant and the two- and three-body masses.

One can introduce an invariant cut-off $\Lambda$ in the integral (4). The requirement that the invariant mass of the two-particle system is smaller than $\Lambda$ (LB regularization), i.e.

$$
M_{20}^{2}<\Lambda^{2},
$$

makes the integral (4) finite. Thus, the integral limits are

$$
\int_{0}^{1} d x^{\prime} \int d^{2} k_{\perp}^{\prime} \rightarrow 2 \pi \int_{x_{\min }^{\prime}}^{x_{\max }^{\prime}} \int_{0}^{k_{\max }^{\prime}} k_{\perp}^{\prime} d k_{\perp}^{\prime}
$$

where

$$
\begin{aligned}
x_{\min }^{\prime} & =\frac{1}{2}\left(1-\sqrt{1-4 m^{2} / \Lambda^{2}}\right), \\
x_{\max }^{\prime} & =\frac{1}{2}\left(1+\sqrt{1-4 m^{2} / \Lambda^{2}}\right), \\
k_{\max }^{\prime 2} & =\Lambda^{2} x^{\prime}\left(1-x^{\prime}\right)-m^{2} .
\end{aligned}
$$

Consequently, $t\left(P_{2}\right) \rightarrow t_{\Lambda}\left(P_{2}\right)$ depends on $\Lambda$ as well. The LB regularization scheme has been utilized in [15] and the subsequent calculations in [21]. Accordingly, one can calculate the pole of the two-particle-propagator determining the bound state mass $M_{2 B}$ for any value of the cut-off $\Lambda$. 


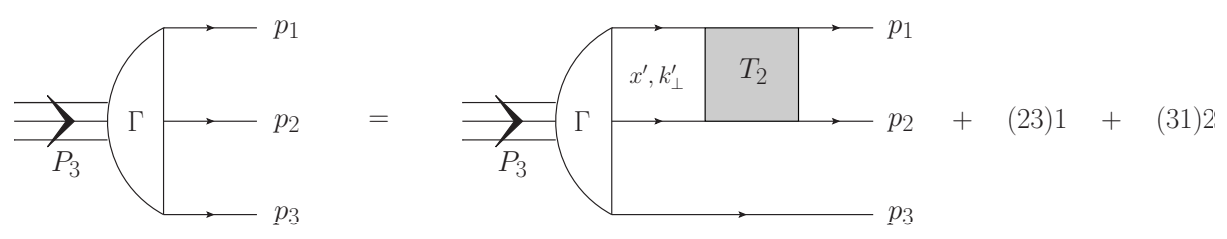

Figure 2: Diagrammatic representation of the Faddeev equation for a zero-range interaction, eq. (15). The gray area indicates the two-body input given in Fig. 1 and eq. (3).

\subsection{The three-body problem}

Investigating the three-body system, we consider the three-body bound state wave function $\Psi(1,2,3)$ on the light front, where $1,2,3$ shorthand for $p_{1}, p_{2}, p_{3}$. The wave function is related to the vertex function $\Gamma$ by

$$
\Psi(1,2,3)=\frac{\Gamma(1,2,3)}{\mathcal{M}^{2}-M_{3 B}^{2}} \quad \text { with } \quad \mathcal{M}^{2}=\left(p_{1}+p_{2}+p_{3}\right)^{2},
$$

where $M_{3 B}$ is the three-body bound state mass. We expand the vertex function $\Gamma$ in the standard way as

$$
\Gamma(1,2,3)=\Gamma_{12}(1,2,3)+\Gamma_{23}(1,2,3)+\Gamma_{31}(1,2,3),
$$

where the Faddeev components $\Gamma_{i j}(1,2,3)$ satisfy equations involving the interaction between particles $(i j)$ only. For three fermions the equation for Faddeev component $\Gamma_{12}(1,2,3)$, by adding the spin indices, reads

$$
\begin{aligned}
\Gamma_{\sigma_{1} \sigma_{2} \sigma_{3}}^{\sigma}(1,2,3) & =\frac{1}{(2 \pi)^{3}} \int \sum_{\sigma_{1}^{\prime} \sigma_{2}^{\prime}} \mathcal{K}_{\sigma_{1} \sigma_{2}}^{\sigma_{1}^{\prime} \sigma_{2}^{\prime}}\left(1,2 ; 1^{\prime}, 2^{\prime}\right) \frac{d^{2} k_{\perp}^{\prime} d x^{\prime}}{2 x^{\prime}\left(1-x^{\prime}\right)} \frac{1}{M_{20}^{2}-P_{2}^{2}} \\
& \times\left[\Gamma_{\sigma_{1}^{\prime} \sigma_{2}^{\prime} \sigma_{3}}^{\sigma}\left(1^{\prime}, 2^{\prime}, 3\right)+\Gamma_{\sigma_{2}^{\prime} \sigma_{3} \sigma_{1}^{\prime}}^{\sigma}\left(2^{\prime}, 3,1^{\prime}\right)+\Gamma_{\sigma_{3} \sigma_{1}^{\prime} \sigma_{2}^{\prime}}^{\sigma}\left(3,1^{\prime}, 2^{\prime}\right)\right]
\end{aligned}
$$

where $\sigma$ is the spin projection of the bound state. A diagrammatic representation of this equation is given in Fig. 2. In general, as explained in Appendix A, the arguments of $\Gamma_{\sigma_{1} \sigma_{2} \sigma_{3}}^{\sigma}(1,2,3)$ can be rewritten in variables $\vec{k}_{i \perp}$ and $x_{i}(i=1,2,3)$, where the transversal momenta are $\vec{k}_{i \perp}=\vec{p}_{i \perp}-x_{i} \vec{P}_{3 \perp}$ and the fraction of the total plus component carried by the single particles is given by $x_{i}=p_{i}^{+} / P_{3}^{+}$. Here $P_{3}^{\mu}$ denotes the momentum of the three-body system. Alternatively, one can rearrange the now defined variables of two 
particles $i$ and $j$ to the corresponding pair variables $\vec{k}_{\perp}$ and $x$ introduced for the two-body problem. This is very useful for eq. (15), where a natural split to a pair ( $i j)$ and to a spectator particle (the third one) emerges. The expression for the two-body mass embedded in the three-body system is

$$
\begin{aligned}
P_{2}^{2} & =\left(P_{3}-p_{3}\right)^{2} \\
& =\left(M_{3}-p_{3}^{+}\right)\left(M_{3}-\frac{\vec{p}_{3 \perp}^{2}+m^{2}}{p_{3}^{+}}\right)-\vec{p}_{3 \perp}^{2},
\end{aligned}
$$

where the momentum of the three-body system is taken at rest, i.e. $P_{3}^{\mu}=$ $\left(M_{3}, M_{3}, 0,0\right)$ in standard light front coordinates. Using the variables introduced the following relation

$$
P_{2}^{2}=\left(1-x_{3}\right) M_{3}^{2}-\frac{\vec{k}_{3 \perp}^{2}+\left(1-x_{3}\right) m^{2}}{x_{3}},
$$

holds.

In this work we investigate a bound state with total spin $\sigma=1 / 2$. Because of the factorized form of the kernels, the Faddeev component $\Gamma_{\sigma_{1} \sigma_{2} \sigma_{3}}^{\sigma}(1,2,3)$ must be proportional to $\bar{K}_{\sigma_{1} \sigma_{2}}(1,2)$ and hence has the form

$$
\Gamma_{\sigma_{1} \sigma_{2} \sigma_{3}}^{\sigma}(1,2,3)=\bar{K}_{\sigma_{1} \sigma_{2}}(1,2) G_{\sigma_{3}}^{\sigma}(1,2,3) .
$$

Using eq.(15) one finds the equation for $G_{\sigma_{3}}^{\sigma}(1,2,3)$

$$
\begin{aligned}
G_{\sigma_{3}}^{\sigma}(1,2,3) & =\frac{\lambda}{(2 \pi)^{3}} \int \sum_{\sigma_{1}^{\prime} \sigma_{2}^{\prime}} K_{\sigma_{1} \sigma_{2}}^{\sigma_{1}^{\prime} \sigma_{2}^{\prime}}\left(1,2 ; 1^{\prime}, 2^{\prime}\right) \frac{d^{2} k_{\perp}^{\prime} d x^{\prime}}{2 x^{\prime}\left(1-x^{\prime}\right)} \frac{1}{M_{20}^{2}-P_{2}^{2}} \\
& \times\left[\bar{K}_{\sigma_{1}^{\prime} \sigma_{2}^{\prime}}\left(1^{\prime}, 2^{\prime}\right) G_{\sigma_{3}}^{\sigma}\left(1^{\prime}, 2^{\prime}, 3\right)+\bar{K}_{\sigma_{2}^{\prime} \sigma_{3}}\left(2^{\prime}, 3\right) G_{\sigma_{1}^{\prime}}^{\sigma}\left(2^{\prime}, 3,1^{\prime}\right)\right. \\
& \left.+\bar{K}_{\sigma_{3} \sigma_{1}^{\prime}}\left(3,1^{\prime}\right) G_{\sigma_{2}^{\prime}}^{\sigma}\left(3,1^{\prime}, 2^{\prime}\right)\right]
\end{aligned}
$$

The functions $G_{\sigma_{3}}^{\sigma}$ are the components of a $2 \times 2$ matrix labeled by the spin indices. In order to decompose $G_{\sigma_{3}}^{\sigma}$ into basis structures we follow Refs. [22, 23], i.e. represent it as a projection to spin states of four independent elements from the Dirac space. Introducing the orthogonal $4 \times 4$ matrices $\left\{S_{i}\right\}$ the matrix $G_{\sigma_{3}}^{\sigma}$ can be written as

$$
G_{\sigma_{3}}^{\sigma}(1,2,3)=\sum_{i=1}^{4} g_{i}(1,2,3) \bar{u}_{\sigma_{3}}\left(p_{3}\right) S_{i} u^{\sigma}\left(P_{3}\right) .
$$


The explicit formulae for the $\left\{S_{i}\right\}$ are given in the Appendix B. Note that the basis elements satisfy the following orthogonality conditions

$$
\operatorname{Tr}\left[\bar{S}_{i}\left(\not p_{3}+m\right) S_{j}\left(\not p_{3}+M_{3}\right)\right]=0 \quad \text { for } \quad i \neq j
$$

with $\bar{S}_{i}=\gamma_{0} S_{i}^{\dagger} \gamma_{0}$. The matrices are not normalized to one rather

$$
\operatorname{Tr}\left[\bar{S}_{i}\left(\not p_{3}+m\right) S_{i}\left(\not p_{3}+M_{3}\right)\right]=N_{i}= \begin{cases}8 x_{3} k_{\perp}^{2} & \text { for } i=1 \\ 8 x_{3} m^{2} & \text { for } i=2, \\ 8 x_{3} k_{\perp}^{2} C_{\mathrm{ps}}^{2} & \text { for } i=3 \\ 8 x_{3} m^{2} C_{\mathrm{ps}}^{2} & \text { for } i=4\end{cases}
$$

The factors $C_{\mathrm{ps}}$ and $C_{\mathrm{ps}}^{2}$ are given by [24]

$$
C_{\mathrm{ps}}=\frac{1}{m^{2} \omega \cdot P_{3}} \varepsilon^{\mu \nu \rho \gamma} p_{1_{\mu}} p_{2_{\nu}} P_{3 \rho} \omega_{\gamma}, \quad C_{\mathrm{ps}}^{2}=\frac{1}{m^{4}}\left[k_{3 \perp}^{2} k_{\perp}^{2}-\left(\vec{k}_{3 \perp} \cdot \vec{k}_{\perp}\right)^{2}\right],
$$

where $\omega$ is a four-vector with $\omega^{2}=0$ determining the light front orientation by the equation $\omega \cdot x=0$. We use $\omega=(1,0,0,-1)$ to recover the standard LF approach. We keep $\omega$ unspecified in the formulae (23) for shortness. Observe that the matrix $\bar{K}_{\sigma_{1} \sigma_{2}}(1,2)$ in eq. (18) is antisymmetric with respect to the permutation $1 \leftrightarrow 2$. The functions $g_{1,2}$ are symmetric, i.e. $g_{1,2}(1,2,3)=g_{1,2}(2,1,3)$. On the other hand the function $g_{3,4}$ are antisymmetric, i.e. $g_{3,4}(1,2,3)=-g_{3,4}(2,1,3)$, because of the additional antisymmetric factor $C_{\mathrm{ps}}$. Substitution of the decomposition (20) into eq. (19) leads to

$$
\begin{aligned}
N_{i} g_{i}(1,2,3) & =\frac{\lambda}{(2 \pi)^{3}} \int \frac{d^{2} k_{\perp}^{\prime} d x^{\prime}}{2 x^{\prime}\left(1-x^{\prime}\right)} \frac{1}{M_{20}^{2}-P_{2}^{2}} \\
& \times\left[K N_{i}^{\prime} g_{i}\left(1^{\prime}, 2^{\prime}, 3\right)+\sum_{j=1} v_{i j}^{b} g_{j}\left(2^{\prime}, 3,1^{\prime}\right)+\sum_{j=1}^{4} v_{i j}^{c} g_{j}\left(3,1^{\prime}, 2^{\prime}\right)\right]
\end{aligned}
$$

The factors $N_{1,2}^{\prime}$ are the same as $N_{1,2}$, whereas in $N_{3,4}^{\prime}$ the factor $C_{\mathrm{ps}}^{2}$ is replaced by

$$
\begin{aligned}
C_{\mathrm{ps}} C_{\mathrm{ps}}^{\prime} & =\left(\frac{1}{m^{2} \omega \cdot P_{3}}\right)^{2} \varepsilon^{\mu \nu \rho \gamma} p_{1_{\mu}} p_{2_{\nu}} P_{3 \rho} \omega_{\gamma} \varepsilon^{\bar{\mu} \bar{\nu} \bar{\rho} \bar{\gamma}} p_{1_{\bar{\mu}}}^{\prime} p_{2_{\bar{\nu}}}^{\prime} P_{3 \bar{\rho}} \omega_{\bar{\gamma}} \\
& =\frac{1}{m^{4}}\left(\left(\vec{k}_{\perp}^{\prime} \cdot \vec{k}_{\perp}\right) k_{3 \perp}^{2}-\left(\vec{k}_{\perp} \cdot \vec{k}_{3 \perp}\right)\left(\vec{k}_{\perp}^{\prime} \cdot \vec{k}_{3 \perp}\right)\right) .
\end{aligned}
$$


Furthermore the kernels $v_{i j}^{b}$ and $v_{i j}^{c}$ are given by

$$
\begin{aligned}
v_{i j}^{b} & =\sum \bar{u}_{\sigma}\left(P_{3}\right) \bar{S}_{i} u^{\sigma_{3}}\left(p_{3}\right) K^{\sigma_{1}^{\prime} \sigma_{2}^{\prime}}\left(1^{\prime}, 2^{\prime}\right) \bar{K}_{\sigma_{2}^{\prime} \sigma_{3}}\left(2^{\prime}, 3\right) \bar{u}_{\sigma_{1}^{\prime}}\left(p_{1}^{\prime}\right) S_{b j}^{\prime} u^{\sigma}\left(P_{3}\right), \\
v_{i j}^{c} & =\sum \bar{u}_{\sigma}\left(P_{3}\right) \bar{S}_{i} u^{\sigma_{3}}\left(p_{3}\right) K^{\sigma_{1}^{\prime} \sigma_{2}^{\prime}}\left(1^{\prime}, 2^{\prime}\right) \bar{K}_{\sigma_{3} \sigma_{1}^{\prime}}\left(3,1^{\prime}\right) \bar{u}_{\sigma_{2}^{\prime}}\left(p_{2}^{\prime}\right) S_{c j}^{\prime} u^{\sigma}\left(P_{3}\right) .
\end{aligned}
$$

In Appendix B we explicitly explain how the components of $S_{b}^{\prime}$ and $S_{c}^{\prime}$ are constructed. The factorization of the contact kernel ensures that $g_{3}=g_{4}=0$, as shown in Ref. [22]. Similarly one proofs that $g_{1}$ and $g_{2}$ depend on $\vec{k}_{3 \perp}$ and $x_{3}$ only, which we rename to $\vec{q}_{\perp}$ and $y$ respectively. Therefore the contribution of $v_{i j}^{b}$ and $v_{i j}^{c}$ are equal to each other. Additionally, since the first term $g_{i}$ on the r.h.s. of eq. (24) does not depend on the integration variables, we can rearrange the integral equation in terms of the two-particle propagator $t\left(P_{2}\right)$ given in eqs.(3)-(4). The general form of the integral equations for the three-body bound state reads

$$
\begin{aligned}
& g_{1}\left(y, \vec{q}_{\perp}\right)=\frac{t\left(P_{2}\right)}{N_{1}(2 \pi)^{3}} \int_{0}^{1} d x^{\prime} \int d^{2} k_{\perp}^{\prime} \frac{v_{11} g_{1}\left(x^{\prime}(1-y), \vec{k}_{\perp}^{\prime}\right)+v_{12} g_{2}\left(x^{\prime}(1-y), \vec{k}_{\perp}^{\prime}\right)}{\left(\vec{k}_{\perp}^{\prime}+x^{\prime} \vec{q}_{\perp}\right)^{2}+m^{2}-x^{\prime}\left(1-x^{\prime}\right) P_{2}^{2}} \\
& g_{2}\left(y, \vec{q}_{\perp}\right)=\frac{t\left(P_{2}\right)}{N_{2}(2 \pi)^{3}} \int_{0}^{1} d x^{\prime} \int d^{2} k_{\perp}^{\prime} \frac{v_{21} g_{1}\left(x^{\prime}(1-y), \vec{k}_{\perp}^{\prime}\right)+v_{22} g_{2}\left(x^{\prime}(1-y), \vec{k}_{\perp}^{\prime}\right)}{\left(\vec{k}_{\perp}^{\prime}+x^{\prime} \vec{q}_{\perp}\right)^{2}+m^{2}-x^{\prime}\left(1-x^{\prime}\right) P_{2}^{2}} .
\end{aligned}
$$

Finally, replacing $x^{\prime}(1-y) \rightarrow x^{\prime}$ the equations (27) is transformed to

$$
\begin{aligned}
& g_{1}\left(y, \vec{q}_{\perp}\right)=\frac{t\left(P_{2}\right)}{N_{1}(2 \pi)^{3}} \int_{0}^{1-y} d x^{\prime} \int d^{2} k_{\perp}^{\prime} \frac{v_{11} g_{1}\left(x^{\prime}, \vec{k}_{\perp}^{\prime}\right)+v_{12} g_{2}\left(x^{\prime}, \vec{k}_{\perp}^{\prime}\right)}{P_{3}^{2}-M_{30}^{2}}, \\
& g_{2}\left(y, \vec{q}_{\perp}\right)=\frac{t\left(P_{2}\right)}{N_{2}(2 \pi)^{3}} \int_{0}^{1-y} d x^{\prime} \int d^{2} k_{\perp}^{\prime} \frac{v_{21} g_{1}\left(x^{\prime}, \vec{k}_{\perp}^{\prime}\right)+v_{22} g_{2}\left(x^{\prime}, \vec{k}_{\perp}^{\prime}\right)}{P_{3}^{2}-M_{30}^{2}},
\end{aligned}
$$

where $M_{30}^{2}$ is the mass square of the virtual three-particle state in the rest system, i.e.

$$
M_{30}^{2}=\frac{\vec{k}_{\perp}^{2}+m^{2}}{x^{\prime}}+\frac{\vec{q}_{\perp}^{2}+m^{2}}{y}+\frac{\left(\vec{k}^{\prime}+\vec{q}\right)_{\perp}^{2}+m^{2}}{1-x^{\prime}-y} .
$$

In order to consistently perform the LB regularization in the two- as well in the three-fermion equations we also constrain the mass square of the virtual three-particle state by

$$
\int_{0}^{1-y} d x^{\prime} \int d^{2} k_{\perp}^{\prime} \rightarrow \int_{0}^{1-y} d x^{\prime} \int d^{2} k_{\perp}^{\prime} \theta\left(M_{30}^{2}-\Lambda^{2}\right) .
$$


After fixing an explicit interaction kernel and spin structure the aim is to solve the eigenvalue problem posed by eqs. (28) in order to obtain the mass $M_{3}^{2}=P_{3}^{2}$ of the three-fermion system as a function of the the coupling constant $\lambda$.

\section{The ${ }^{1} S_{0}$ Model}

In this section we investigate a particular kernel which leads to significant simplifications in the three-body equations (28). The spin structure of this kernel is given by

$$
\begin{aligned}
\bar{K}_{\sigma_{1} \sigma_{2}}(1,2) & =\frac{m}{\omega \cdot\left(p_{1}+p_{2}\right)}\left[\bar{u}_{\sigma_{1}}\left(p_{1}\right) i \psi \gamma_{5} U_{\mathrm{c}} \bar{u}_{\sigma_{2}}\left(p_{2}\right)\right], \\
K_{\sigma_{1}^{\prime} \sigma_{2}^{\prime}}\left(1^{\prime}, 2^{\prime}\right) & =\frac{m}{\omega \cdot\left(p_{1}+p_{2}\right)}\left[u^{\sigma_{2}^{\prime}}\left(p_{2}^{\prime}\right) U_{\mathrm{c}} i \psi \gamma_{5} u^{\sigma_{1}^{\prime}}\left(p_{1}^{\prime}\right)\right],
\end{aligned}
$$

where $U_{\mathrm{c}}=i \gamma^{0} \gamma^{2}$ denotes the charge conjugation operator. In the nonrelatististic limit the kernel reads

$$
\begin{aligned}
\mathcal{K}_{\sigma_{1} \sigma_{2}}^{\sigma_{1}^{\prime} \sigma_{2}^{\prime}}\left(1,2 ; 1^{\prime}, 2^{\prime}\right) & \propto \frac{\lambda}{2}\left(w_{\sigma_{1}}^{*} i \sigma_{y} w_{\sigma_{2}}^{*}\right)\left(w_{\sigma_{2}^{\prime}} i \sigma_{y} w_{\sigma_{1}^{\prime}}\right) \\
& =\lambda C_{(1 / 2) \sigma_{1}(1 / 2) \sigma_{2}}^{00} C_{(1 / 2) \sigma_{1}^{\prime}(1 / 2) \sigma_{2}^{\prime}}^{00}\left(w_{\sigma_{1}}^{*} i \sigma_{y} w_{\sigma_{2}}^{*}\right)\left(w_{\sigma_{2}^{\prime}} i \sigma_{y} w_{\sigma_{1}^{\prime}}\right)
\end{aligned}
$$

where $w_{\sigma}$ represent the two-component (Pauli) spinors and $C_{(1 / 2) \sigma_{1}(1 / 2) \sigma_{2}}^{00}$ are the Clebsh-Gordon coefficients [25]. Therefore, this kernel corresponds to an interaction in the ${ }^{1} \mathrm{~S}_{0}$ state.

For this interaction we find

$$
\begin{aligned}
K & =\lambda^{-1} \operatorname{Tr}\{\mathcal{K}\} \\
& =\frac{m^{2}}{\left(\omega \cdot P_{2}\right)^{2}} \sum_{\sigma_{1}^{\prime} \sigma_{2}^{\prime}}\left[u^{\sigma_{2}^{\prime}}\left(p_{2}^{\prime}\right) U_{\mathrm{c}} i \psi \gamma_{5} u^{\sigma_{1}^{\prime}}\left(p_{1}^{\prime}\right)\right]\left[\bar{u}_{\sigma_{1}^{\prime}}\left(p_{1}^{\prime}\right) i \psi \gamma_{5} U_{\mathrm{c}} \bar{u}_{\sigma_{2}^{\prime}}\left(p_{2}^{\prime}\right)\right] \\
& =8 \lambda m^{2} x(1-x) .
\end{aligned}
$$

From equation (4) the loop integral $B_{2}\left(P_{2}\right)$ reads

$$
B\left(P_{2}\right)=-i \frac{4 m^{2}}{(2 \pi)^{3}} \int d x^{\prime} d^{2} k_{\perp}^{\prime} \frac{1}{P_{2}^{2}-M_{20}^{2}} .
$$

Using the $M_{2 B}$ regularization one finds

$$
t\left(P_{2}\right)=\frac{48 \pi^{2}}{4 m^{2}} \kappa\left(P_{2}, M_{2 B}\right)
$$


where

$$
\begin{aligned}
\kappa\left(P_{2}, M_{2 B}\right) & =\left[\frac{3 \Upsilon^{2}\left(P_{2}\right)+1}{\Upsilon^{3}\left(P_{2}\right)} \arctan \Upsilon\left(P_{2}\right)-\frac{1}{\Upsilon^{2}\left(P_{2}\right)}\right. \\
& \left.-\frac{3 \Upsilon^{2}\left(M_{2 B}\right)+1}{\Upsilon^{3}\left(M_{2 B}\right)} \arctan \Upsilon\left(M_{2 B}\right)+\frac{1}{\Upsilon^{2}\left(M_{2 B}\right)}\right]^{-1}
\end{aligned}
$$

with

$$
\Upsilon\left(P_{2}\right)=\left\{\begin{array}{ll}
\frac{P_{2}}{\sqrt{4 m^{2}-P_{2}^{2}}} & \text { for } 0 \leq P_{2}^{2} \leq 4 m^{2}, \\
\frac{\sqrt{-P_{2}^{2}}}{\sqrt{4 m^{2}-P_{2}^{2}}} & \text { for } P_{2}^{2}<0
\end{array} .\right.
$$

Introducing the invariant cut-off as in eq.(8) the loop integral can be directly evaluated using the limits of the integration given in eqs.(10)-(12). In this way, for any value of the cut-off and the coupling we can calculate the pole of the two-fermion propagator.

Now we turn to the three-fermion case. Calculating the $2 \times 2$ matrix for the kernel (31) using eq.(26) yields

$$
\begin{aligned}
& v_{11}=32 m^{2} y x^{2}\left(\vec{q}_{\perp}^{2}+\vec{k}_{\perp}^{\prime} \cdot \vec{q}_{\perp}\right), \\
& v_{12}=0, \\
& v_{21}=32 m^{4} y x^{\prime}\left(1-2 x^{\prime}\right), \\
& v_{11}=-32 m^{4} y x^{\prime}\left(1-x^{\prime}\right) .
\end{aligned}
$$

Shifting $x^{\prime}(1-y) \rightarrow x^{\prime}$ and using $z=x^{\prime} /(1-y)$ we find the following set of equations

$$
\begin{aligned}
g_{1}\left(y, \vec{q}_{\perp}\right)= & \frac{4 m^{2} t\left(P_{2}\right)}{(2 \pi)^{3}} \int_{0}^{1-y} \frac{d x^{\prime}}{x^{\prime}\left(1-y-x^{\prime}\right)} \int d^{2} k_{\perp}^{\prime} \theta\left(M_{30}^{2}-\Lambda^{2}\right) \\
& \frac{\left(1+\vec{k}_{\perp}^{\prime} \cdot \vec{q}_{\perp} / \vec{q}_{\perp}^{2}\right) z^{2}}{P_{3}^{2}-M_{30}^{2}} g_{1}\left(x^{\prime}, \vec{k}_{\perp}^{\prime}\right), \\
g_{2}\left(y, \vec{q}_{\perp}\right)= & \frac{4 m^{2} t\left(P_{2}\right)}{(2 \pi)^{3}} \int_{0}^{1-y} \frac{d x^{\prime}}{x^{\prime}\left(1-y-x^{\prime}\right)} \int d^{2} k_{\perp}^{\prime} \theta\left(M_{30}^{2}-\Lambda^{2}\right) \\
& \frac{z(1-2 z) g_{1}\left(x^{\prime}, \vec{k}_{\perp}^{\prime}\right)-z(1-z) g_{2}\left(x^{\prime}, \vec{k}_{\perp}^{\prime}\right)}{P_{3}^{2}-M_{30}^{2}} .
\end{aligned}
$$

The scalar function $g_{2}$ enters in the second equation only. The first equation differs from the three-boson equation by the factor $\left(1+\vec{k}_{\perp}^{\prime} \cdot \vec{q}_{\perp} / \vec{q}_{\perp}^{2}\right) z^{2}$. We point out that the complete system of equations must be solved, not only the first equation as in Ref. [20]. 


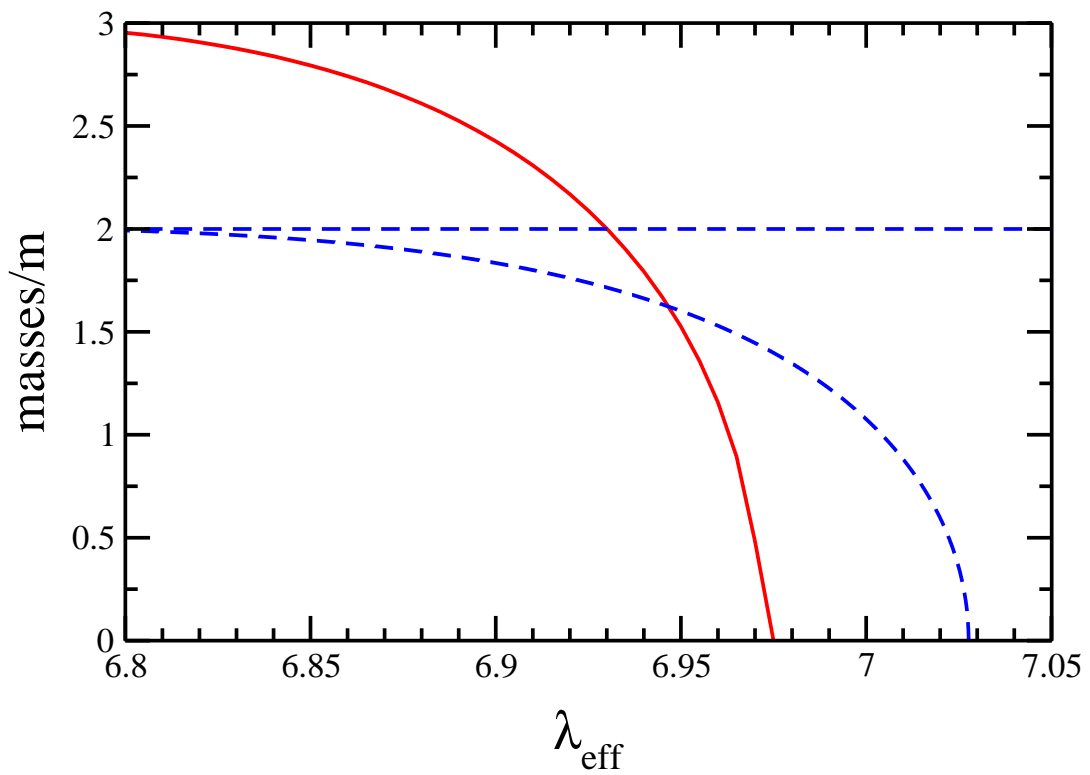

Figure 3: The solution of the two- (dashed) and three-fermion (solid) bound state equations as a function of the effective coupling $\lambda_{\text {eff }}=\lambda m^{2}$. Horizontal dashed line shows the twofermion break-up.

\section{Results}

The solution of the equations (39)-(40) allows us to determine the threefermion mass. In Ref. [20] eq. (39) is solved using the $M_{2 B}$ regularization. Instead we consider the LB regularized version of (39)-(40). In order to compare our results with those of [20] we set the cut-off to a large but finite value, i.e. $\Lambda=10^{15} \mathrm{~m}$, see Ref. [15]. For the bosonic system it was shown by one of the authors that for large invariant cut-off the resulting function $M_{3 B}\left(M_{2 B}\right)$ coincides with the analogous results in $M_{2 B}$ regularization [15, 21]. Therefore, we expect this feature as well in the fermionic case.

Fig. 3 shows the masses $M_{2 B}$ of the two-fermion bound state and $M_{3 B}$ of the three-fermion bound state as a function of the effective coupling strength $\lambda_{\text {eff }}=\lambda m^{2}$. All masses $M_{2 B}$ and $M_{3 B}$ are given in units of the elementary fermion mass $m$.

Note the following features:

(i) The two- as well as the three-fermion mass are monotonic function of the coupling. In particular, for the critical coupling $\lambda_{\text {eff }}^{\mathrm{c}} \approx 6.972$ the 
three-fermion bound state mass vanishes. This is the relativistic analog of the Thomas collapse [13] and is known to occur for the three-boson bound state with a similar zero-range interaction, see e.g. [15, 21]. This critical coupling corresponds to a critical value of two-fermion mass $M_{2 B}^{\mathrm{c}} \approx 1.42 \mathrm{~m}$. In comparison, the critical two-fermion mass $M_{2 B}^{\mathrm{c}} \approx 1.35 \mathrm{~m}$ calculated in [20] is notable lower.

(ii) As $M_{2 B} \rightarrow 2 m$, the three-fermion bound state still exists. This may give rise to the Efimov effect [26, 27], an interesting issue for further investigations.

(iii) There is a region of parameter space where both $M_{2 B}$ and $M_{3 B}$ exist. Only in this case it is possible to plot $M_{3 B}$ against $M_{2 B}$.

We focus on the investigation in the region of the coupling strength $\lambda$, where the two-fermion bound state exists. Therefore, by comparison to the results of [20], we can estimate the role of the coupled structure of (39)-(40). In Fig. 4 the mass $M_{3 B}$ is plotted against $M_{2 B}$. We have also included the solid line for fermions and the dash-dotted line for bosons. For comparison to the calculation of [20] we independently solve, using the LB regularization scheme, eq. (39) solely and present the results in Fig. 4 as dashed line. Furthermore, the dotted line indicates the threshold for the three-body mass.

The resulting functions $M_{3 B}\left(M_{2 B}\right)$ for the bosonic and for the fermionic cases have the same qualitative behavior. By decreasing $M_{2 B}$ the three-body mass decreases very quickly and vanishes at a critical values $M_{2 B}^{\mathrm{c}}$ of the two body mass. The value of the critical mass for the bosonic system is $M_{2 B}^{\mathrm{c}} \approx 1.45 \mathrm{~m}$ and greater than the corresponding values for the fermionic cases, both for the coupled equation system and for the solution of the first equation only.

It is interesting to see why there is this notable discrepancy between the whole calculation and the result obtained by solving (39) alone.

The latter method automatically excludes the trivial solution $g_{1} \equiv 0$ for eq. (39). However, this solution is physically non-trivial in the coupled system.

In more detail, the system of integral equations (39)-(40) can be written as

$$
(I-V)\left(\begin{array}{l}
g_{1} \\
g_{2}
\end{array}\right)=0
$$




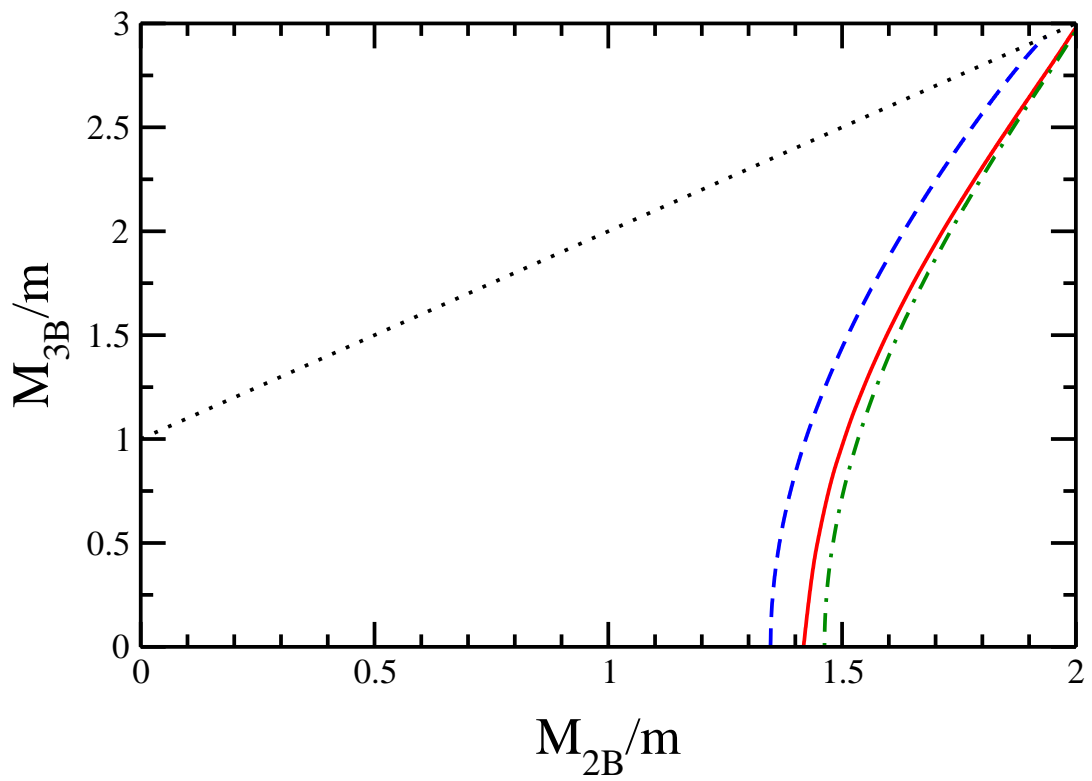

Figure 4: Three-fermion bound state as a function of $M_{2 B}$ (solid line) in comparison to the three-boson bound state (dash-dotted line) and to three-fermion bound state calculated by solving equation (39) alone, with $\Lambda \rightarrow \infty$ (dashed line). To completeness, the threshold for the three-body mass is indicated (dotted line).

where the operator $V$ collects all contributions from $v_{i j}$ and $I$ is the identity operator.

To achieve a numerical solution we have discretized the equations (39)(40) using a momentum space grid $\tau_{k}$, with $k=1, \cdots, n$, where $n$ is the number of the lattice points. Each of the scalar function $g_{i}, i=1,2$ becomes a vector $\tilde{g}_{1}$ of dimension $n$. The operator eigenvalue problem (41) is approximated by the corresponding matrix problem

$$
W \tilde{g}=0 \quad \text { with } \quad \tilde{g}=\left(\begin{array}{c}
\tilde{g}_{1} \\
\tilde{g}_{2}
\end{array}\right),
$$

where

$$
W=\left(\begin{array}{ll}
W_{11} & W_{12} \\
W_{21} & W_{22}
\end{array}\right) .
$$

All possible solutions fulfill of course the condition $\operatorname{det}(W)=0$. For the ${ }^{1} S_{0}$ model (31) one has $W_{12}=0$ and the solvability condition reads

$$
\operatorname{det}(W)=\operatorname{det}\left(W_{11}\right) \cdot \operatorname{det}\left(W_{22}\right)=0 .
$$




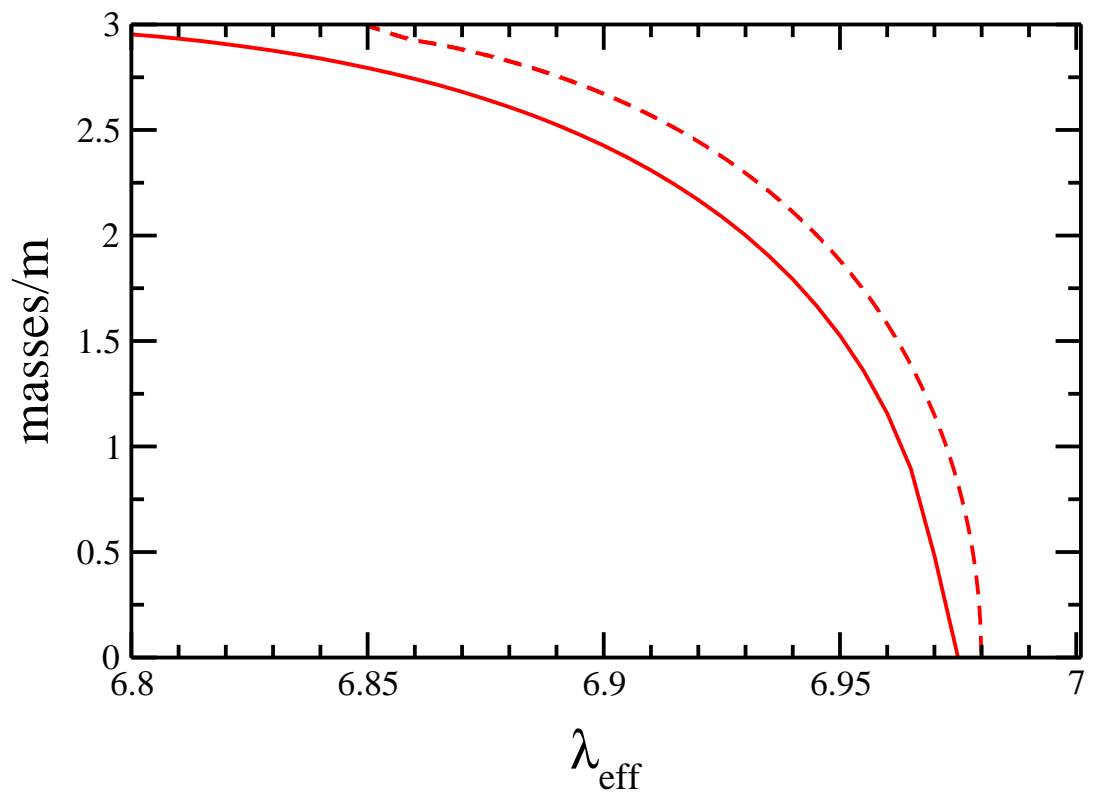

Figure 5: Mass of the three-fermion ground state (solid line) and of the first three-fermion excited state (dashed line) calculated by solving the equation system (39)-(40) as a function of the effective strength $\lambda_{\text {eff }}$.

Evidently, a solution of the whole system satisfies $\operatorname{det}\left(W_{11}\right)=0$ or $\operatorname{det}\left(W_{22}\right)=$ 0 , whereas the solutions of eq.(39) satisfies $\operatorname{det}\left(W_{11}\right)=0$ only. Hence, solving solely eq.(39) the physical solutions corresponding to the case $g_{1} \equiv 0$ can be lost.

Because for arbitrary coupling strength $\lambda$ there exist more solutions to (42), we consider the two smallest eigenvalues. In Fig. 5 the mass of the ground state (lowest eigenstate) and the first excited state (next-to-lowest eigenstate) depending on effective coupling strength $\lambda_{\text {eff }}$ are plotted as solid and dashed line, respectively. The ground state coincides with the solution shown in Fig. 3. For the first excited state we find that the Thomas collapse occurs at $\lambda_{\text {eff }} \approx 6.98$ that corresponds to a critical two-fermion mass $M_{2 B}^{\mathrm{c}} \approx 1.35 \mathrm{~m}$ which is in agreement with the critical value obtained by solving eq.(39) only. We therefore suspect that the solution given in Ref. [20] describes the first excited state. This observation is confirmed in Fig. 6 where the bound state mass solving only eq. (39) (crosses) is compared with the solution for the first excited state (circles) obtained by solving the cou- 


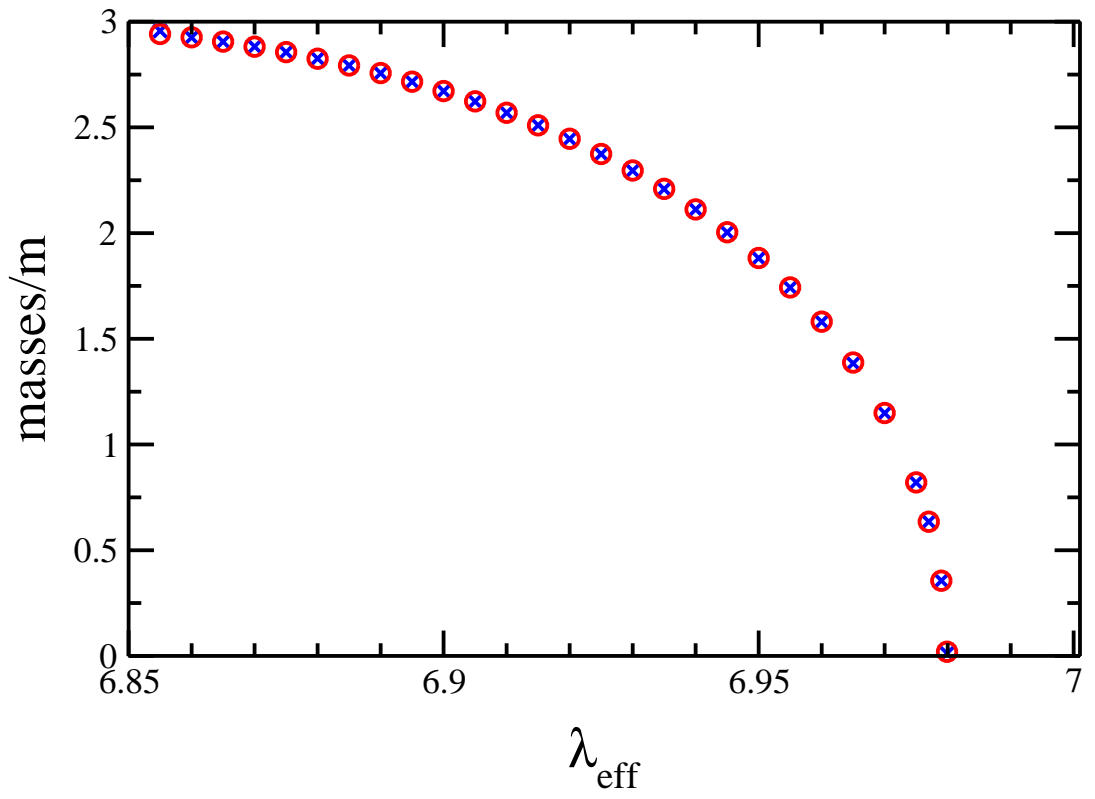

Figure 6: Mass of the three-fermion bound state calculated by solving eq.(39) only (crosses) and mass of the first three-fermion excited state calculated by solving the set of equations (39)-(40) (circles) as a function of the effective strength $\lambda_{\text {eff }}$.

pled equation system (39)-(40) for different values of the effective coupling strength $\lambda_{\text {eff }}$. The two masses agree well for all $\lambda_{\text {eff }}$ but small derivations are noticeable close to the breakup, i.e. for $\lambda_{\text {eff }}<6.86$. In other words the solution of eq.(39) describes three-fermion bound states, but the ground state is missing. The true ground state is only obtained in the solution of the coupled system of integral equations.

\section{Conclusions}

We have derived and solved the coupled equations of a relativistic three fermion system subject to an effective scalar zero-rage interaction in the ${ }^{1} S_{0}$ channel. In the computation of the three- and two-fermion bound states as function of the coupling constant $\lambda$ we have introduced an invariant cut-off $\Lambda$ (LB regularization scheme) and solved the equations for large $\Lambda$. Additionally, we have investigated the solution of eq. (39) alone with the same regularization scheme. Thereby the results of Ref. [20] were confirmed. Analogous to the three-boson system we find the relativistic Thomas collapse 
in both calculations. In particular, solving the coupled equations we find that the two-fermion critical mass $M_{2 B}^{\mathrm{c}} \approx 1.42 \mathrm{~m}$ is different from the value given in [20]. This is explained by the fact that in the computation of Carbonell/Karmanov the lowest three-fermion mass is missing. Instead of the ground state their computed lowest mass seems to agree with the mass of the first excited state. Taking in account the full equation system, we have obtained a critical two-body mass different from the one in the literature and more close to the critical value in the bosonic case (see Fig. 4).

In particular, our results provide evidence we that the bosonic or fermionic nature of the constituents does not play a very important role in this model. Consequently, using the bosonic-type Faddeev equations to describe the isolated nucleon [15] or embedded in a quark matter medium [28, 29] can be justified a posteriori.

Nevertheless, an implementation of the spin degrees of freedom is desirable and a first consistent step in this direction is studied here. Further investigations of the three-fermion correlations in hot and dense quark matter following [29] can be performed using the interaction kernel (31).

The theoretical methods presented here are model independent and allow the investigation of the nucleon with more complicated kernels, such as the one corresponding to an interaction in the $P$-wave state with $S=1$ [22].

Acknowledgment: The authors are grateful to V.A. Karmanov for the providing his theoretical notes [22]. This work is supported by Deutsche Forschungsgemeinschaft and by the Helmholtz International Center for FAIR within the LOEWE program of the State of Hesse.

\section{A. Kinematics}

Let $a^{\mu}=\left(a^{0}, a^{1}, a^{2}, a^{3}\right)$ be a generic four vector. The light front dynamics is given by the transformation

$$
a^{\mu}=\left(a^{+}, a^{-}, a^{1}, a^{2}\right)=\left(a^{+}, a^{-}, \vec{a}_{\perp}\right)
$$

where

$$
a^{ \pm} \equiv a^{0} \pm a^{3} .
$$

The covariant components follow from $a_{\mu}=g_{\mu \nu} a^{\nu}$, where the metric 
tensor is given as,

$$
g_{\mu \nu}=\left(\begin{array}{cccc}
0 & \frac{1}{2} & 0 & 0 \\
\frac{1}{2} & 0 & 0 & 0 \\
0 & 0 & -1 & 0 \\
0 & 0 & 0 & -1
\end{array}\right), g^{\mu \nu}=\left(\begin{array}{cccc}
0 & 2 & 0 & 0 \\
2 & 0 & 0 & 0 \\
0 & 0 & -1 & 0 \\
0 & 0 & 0 & -1
\end{array}\right) .
$$

The mass shell condition for a particle of mass $m$ and momentum $p$ reads

$$
p^{+} p^{-}=\vec{p}_{\perp}^{2}+m^{2} .
$$

For the description of the kinematics of two- and three-particle systems it is convenient to introduce the total momentum of the particles and some internal variables. For two particles with momenta $p_{1}$ and $p_{2}$ and masses $m_{1}$ and $m_{2}$ respectively we define

$$
\begin{aligned}
P_{2} & =p_{1}+p_{2}, \\
x & =p_{1}^{+} / P_{2}^{+}, \quad 1-x=p_{2}^{+} / P_{2}^{+}, \\
k & =(1-x) p_{1}-x p_{2} .
\end{aligned}
$$

The invariant energy square, $s_{12}$, i.e. the mass of the virtual two-body state, is given by

$$
\begin{aligned}
s_{12} \equiv M_{20}^{2} & =P_{2}^{+} P_{2}^{-}-\vec{P}_{2 \perp}^{2}=\sum_{i=1}^{2} \frac{\vec{p}_{i \perp}^{2}+m_{i}^{2}}{k_{i}^{+}} P_{2}^{+}-\vec{P}_{2 \perp}^{2} \\
& =\frac{\vec{p}_{1 \perp}^{2}+m_{1}^{2}}{x}+\frac{\vec{p}_{2 \perp}^{2}+m_{2}^{2}}{1-x}-\vec{P}_{2 \perp}^{2} \\
& =\frac{m_{2}^{2}}{1-x}+\frac{m_{1}^{2}}{x}+\frac{\vec{k}_{\perp}^{2}}{x(1-x)}
\end{aligned}
$$

Choosing $\vec{P}_{2 \perp}=0$ and for equal masses, i.e. $m_{1}=m_{2} \equiv m$ we recover the expression in eq. (5).

For three particles with momenta $p_{1}, p_{2}$ and $p_{3}$ and masses $m_{1}, m_{2}$ and $m_{3}$ we analogously introduce the plus momentum fractions and the total momentum as

$$
\begin{aligned}
& P_{3}=p_{1}+p_{2}+p_{3} \\
& x_{i}=p_{i}^{+} / P_{3}^{+}, \quad \sum_{i=1}^{3} x_{i}=1 .
\end{aligned}
$$


In general one can introduce Jacobi coordinates to treat the kinematics of the three-body system [30]. However, because of the structure of the Faddeevequation, where a spectator particle and a pair of two particles emerge, we prefer to express the kinematics using the variable of the spectator particle -say the third particle - and the one of the pair (12), where kinematics has been discussed above. Note that the momentum fraction of the three-body system $x_{i}$ and the variable $x$ do not coincide but are related by

$$
\begin{aligned}
& x_{1}=x\left(1-x_{3}\right), \\
& x_{2}=(1-x)\left(1-x_{3}\right) .
\end{aligned}
$$

The additional constraint used in this paper, namely that the three-body system is at rest, allows us to connect the single transverse momenta of the particle in the pair with the relative transverse momentum $\vec{k}_{\perp}$ by

$$
\begin{aligned}
& \vec{p}_{1 \perp}=-\vec{k}_{\perp}-x \vec{p}_{3 \perp}, \\
& \vec{p}_{2 \perp}=\vec{k}_{\perp}-(1-x) \vec{p}_{3 \perp} .
\end{aligned}
$$

The invariant energy square, $s_{123}$, i.e. the mass of the virtual three-body state, can be written as

$$
\begin{aligned}
s_{123} \equiv M_{30}^{2} & =P_{3}^{+} P_{3}^{-}-\vec{P}_{3 \perp}^{2}=\sum_{i=1}^{3} \frac{\vec{p}_{i \perp}^{2}+m_{i}^{2}}{k_{i}^{+}} P_{3}^{+}-\vec{P}_{3 \perp}^{2} \\
& =\sum_{i=1}^{3} \frac{\vec{p}_{i \perp}^{2}+m_{i}^{2}}{x_{i}^{+}}-\vec{P}_{3 \perp}^{2} .
\end{aligned}
$$

Demanding $\vec{P}_{3 \perp}=0$ and for equal masses, i.e. $m_{1}=m_{2}=m_{3} \equiv m$ we recover, with the necessary variable identification, eq.(29).

\section{B. Orthogonal basis}

In this section we define the orthogonal basis $\left\{S_{i}\right\}$ as given in Refs. [22, 23] used for the decomposition of the $2 \times 2$-matrix $G_{\sigma_{3}}^{\sigma}$ in eq. (20). We choose 
the form

$$
\begin{aligned}
& S_{1}=\left[2 x_{3}-\left(m+x_{3} M_{3}\right) \frac{\psi}{\omega \cdot P_{3}}\right], \\
& S_{2}=m \frac{\psi}{\omega \cdot P_{3}}, \\
& S_{3}=\left[2 x_{3}-\left(m-x_{3} M_{3}\right) \frac{\psi}{\omega \cdot P_{3}}\right] i C_{\mathrm{ps}} \gamma_{5}, \\
& S_{4}=m \frac{\psi}{\omega \cdot P_{3}} i C_{\mathrm{ps}} \gamma_{5},
\end{aligned}
$$

where the factor $C_{\mathrm{ps}}$ is given in eq.(23).

Furthermore, the matrices $\bar{S}_{i}=\gamma_{0} S_{i}^{\dagger} \gamma_{0}$ explicitly read

$$
\begin{aligned}
& \bar{S}_{1}=\left[2 x_{3}-\left(m+x_{3} M_{3}\right) \frac{\psi}{\omega \cdot P_{3}}\right], \\
& \bar{S}_{2}=m \frac{\psi}{\omega \cdot P_{3}}, \\
& \bar{S}_{3}=i C_{\mathrm{ps}} \gamma_{5}\left[2 x_{3}-\left(m-x_{3} M_{3}\right) \frac{\psi}{\omega \cdot P_{3}}\right], \\
& \bar{S}_{4}=i C_{\mathrm{ps}} \gamma_{5} m \frac{\psi}{\omega \cdot P_{3}} .
\end{aligned}
$$

Note that $\bar{S}_{1,2}=S_{1,2}$. These matrices satisfy the orthogonality (21) and normalization conditions (22).

The permutated structures $S_{b}^{\prime}$ and $S_{c}^{\prime}$ are derived from eq. (64-67). $S_{b}^{\prime}$ is constructed using the permutation $\left(1^{\prime} \rightarrow 2^{\prime} \rightarrow 3 \rightarrow 1^{\prime}\right)$, e.g. for $S_{b 1}^{\prime}$ one performs the substitution $x_{3} \rightarrow x_{1}^{\prime}=(1-x)\left(1-x_{3}\right)$ in eq. (64), while for $S_{b 3}^{\prime}$ and $S_{b 4}^{\prime}$ one additionally has to replace $C_{\mathrm{ps}}$ by

$$
C_{\mathrm{ps}}^{\prime}=\frac{1}{m^{2} \omega \cdot P_{3}} \varepsilon^{\mu \nu \rho \gamma} p_{1_{\mu}}^{\prime} p_{2_{\nu}}^{\prime} P_{3 \rho} \omega_{\gamma} \rightarrow \frac{1}{m^{2} \omega \cdot P_{3}} \varepsilon^{\mu \nu \rho \gamma} p_{2_{\mu}}^{\prime} p_{3_{\nu}} P_{3 \rho} \omega_{\gamma}
$$

in eq. (66) and (67) respectively. Analogously, $S_{c}^{\prime}$ is derived from $S$ by the permutation $\left(1^{\prime} \rightarrow 3 \rightarrow 2^{\prime} \rightarrow 1^{\prime}\right)$, e.g. for $S_{c 1}$ the substitution $x_{3} \rightarrow x_{2}^{\prime}=$ $x\left(1-x_{3}\right)$ has to be used in eq. (64), while for $S_{c 3}^{\prime}$ and $S_{c 4}^{\prime}$ one has to use

$$
C_{\mathrm{ps}}^{\prime}=\frac{1}{m^{2} \omega \cdot P_{3}} \varepsilon^{\mu \nu \rho \gamma} p_{1_{\mu}}^{\prime} p_{2_{\nu}}^{\prime} P_{3 \rho} \omega_{\gamma} \rightarrow \frac{1}{m^{2} \omega \cdot P_{3}} \varepsilon^{\mu \nu \rho \gamma} p_{3_{\mu}} p_{1_{\nu}} P_{3 \rho} \omega_{\gamma}
$$

instead of $C_{\mathrm{ps}}$ in eq. (66) and (67) respectively. 


\section{References}

[1] W. R. B. de Araujo, J. P. B. C. de Melo, T. Frederico, Faddeev null plane model of the nucleon, Phys. Rev. C52 (1995) 2733-2737, doi: 10.1103/PhysRevC.52.2733.

[2] B. D. Keister, W. N. Polyzou, Relativistic Hamiltonian dynamics in nuclear and particle physics, Adv. Nucl. Phys. 20 (1991) 225-479.

[3] R. J. Perry, A. Harindranath, K. G. Wilson, Light front TammDancoff field theory, Phys. Rev. Lett. 65 (1990) 2959-2962, doi: 10.1103/PhysRevLett.65.2959.

[4] I. G. Aznaurian, A. S. Bagdasaryan, N. L. Ter-Isaakian, Relativistic quark model in the infinite momentum frame and static characteristics of nucleons, Phys. Lett. B112 (1982) 393-396, doi:10.1016/03702693(82)91076-0.

[5] Z. Dziembowski, Relativistic model of nucleon; static properties and electromagnetic soft form-factors, Phys. Rev. D37 (1988) 778, doi: 10.1103/PhysRevD.37.778.

[6] P. L. Chung, F. Coester, Relativistic constituent quark model of nucleon form- factors, Phys. Rev. D44 (1991) 229-241, doi: 10.1103/PhysRevD.44.229.

[7] L. D. Faddeev, Scattering theory for a three particle system, Sov. Phys. JETP 12 (1961) 1014-1019.

[8] T. Frederico, Null plane model of three bosons with zero range interaction, Phys. Lett. B282 (1992) 409-414, doi:10.1016/0370-2693(92)90661M.

[9] D. V. Fedorov, A. S. Jensen, Regularized zero-range model and an application to the triton and the hypertriton, Nucl. Phys. A697 (2002) 783-801, doi:10.1016/S0375-9474(01)01266-0.

[10] D. V. Fedorov, A. S. Jensen, Correlation induced collapse of many-body systems with zero-range potentials, Phys. Rev. A63 (2001) 063608, doi: 10.1103/PhysRevA.63.063608. 
[11] J. Carbonell, V. A. Karmanov, Three-boson relativistic bound states with zero-range interaction, Phys. Rev. C67 (2003) 037001, doi: 10.1103/PhysRevC.67.037001.

[12] M. T. Yamashita, T. Frederico, L. Tomio, Neutron $-{ }^{19} \mathrm{C}$ scattering near an Efimov state, Phys. Lett. B670 (2008) 49-54, doi: 10.1016/j.physletb.2008.10.030.

[13] L. H. Thomas, The Interaction Between a Neutron and a Proton and the Structure of $\mathrm{H}^{* *} 3$, Phys. Rev. 47 (1935) 903-909, doi: 10.1103/PhysRev.47.903.

[14] G. P. Lepage, S. J. Brodsky, Exclusive Processes in Perturbative Quantum Chromodynamics, Phys. Rev. D22 (1980) 2157, doi: 10.1103/PhysRevD.22.2157.

[15] M. Beyer, S. Mattiello, T. Frederico, H. J. Weber, On the stability of three-body bound states on the light front, Few Body Syst. 33 (2003) 89-97, doi:10.1007/s00601-003-0014-2.

[16] W. R. B. de Araujo, E. F. Suisso, T. Frederico, M. Beyer, H. J. Weber, Relativistic quark spin coupling effects in the nucleon electromagnetic form factors, Phys. Lett. B478 (2000) 86-93, doi:10.1016/S03702693(00)00238-0.

[17] W. R. B. de Araujo, T. Frederico, M. Beyer, H. J. Weber, Neutron charge radius: Relativistic effects and the Foldy term, Int. J. Mod. Phys. A18 (2003) 5767-5780, doi:10.1142/S0217751X03015866.

[18] E. F. Suisso, W. R. B. de Araujo, T. Frederico, M. Beyer, H. J. Weber, Relativistic quark spin coupling effects in the correlations between nucleon electroweak properties, Nucl. Phys. A694 (2001) 351-371, doi: 10.1016/S0375-9474(01)00978-2.

[19] W. R. B. de Araujo, T. Frederico, M. Beyer, H. J. Weber, mu(p) $\mathrm{G}(\mathrm{E}(\mathrm{p})) / \mathrm{G}(\mathrm{M}(\mathrm{p}))$ and $\mathrm{qF} 2(\mathrm{p}) / \mathrm{F} 1(\mathrm{p})$ in a relativistic quark model, Eur. Phys. J. A29 (2006) 227-234, doi:10.1140/epja/i2006-10072-2.

[20] V. A. Karmanov, J. Carbonell, Critical stability of three-body relativistic bound states with zero-range interaction, Few Body Syst. 34 (2004) 85-90. 
[21] S. Mattiello, The stability of the relativistic three-body system and inmedium equations, Few Body Syst. 34 (2004) 119-125.

[22] V. A. Karmanov, Three-fermion relativistic bound states with zerorange interaction, private communication (2004) .

[23] V. A. Karmanov, J. F. Mathiot, A. V. Smirnov, Nonperturbative calculation of the anomalous magnetic moment in the Yukawa model within truncated Fock space, Phys. Rev. D82 (2010) 056010, doi: 10.1103/PhysRevD.82.056010.

[24] V. A. Karmanov, The nucleon wave function in light-front dynamics, Nucl. Phys. A644 (1998) 165-200, doi:10.1016/S0375-9474(98)00579-X.

[25] J. Carbonell, B. Desplanques, V. A. Karmanov, J. F. Mathiot, Explicitly covariant light-front dynamics and relativistic few-body systems, Phys. Rept. 300 (1998) 215-347, doi:10.1016/S0370-1573(97)00090-2.

[26] V. Efimov, Energy levels arising form the resonant two-body forces in a three-body system, Phys. Lett. B33 (1970) 563-564.

[27] E. Braaten, H. W. Hammer, Universality in Few-body Systems with Large Scattering Length, Phys. Rept. 428 (2006) 259-390, doi: 10.1016/j.physrep.2006.03.001.

[28] M. Beyer, S. Mattiello, T. Frederico, H. J. Weber, Three-quark clusters at finite temperatures and densities, Phys. Lett. B521 (2001) 33-41, doi:10.1016/S0370-2693(01)01175-3.

[29] S. Strauss, S. Mattiello, M. Beyer, Light-front Nambu-Jona-Lasinio model at finite temperature and density, J. Phys. G36 (2009) 085006, doi:10.1088/0954-3899/36/8/085006.

[30] B. L. G. Bakker, L. A. Kondratyuk, M. V. Terentev, On the formulation of two-body and three-body relativistic equations employing light front dynamics, Nucl. Phys. B158 (1979) 497, doi:10.1016/05503213(79)90179-2. 